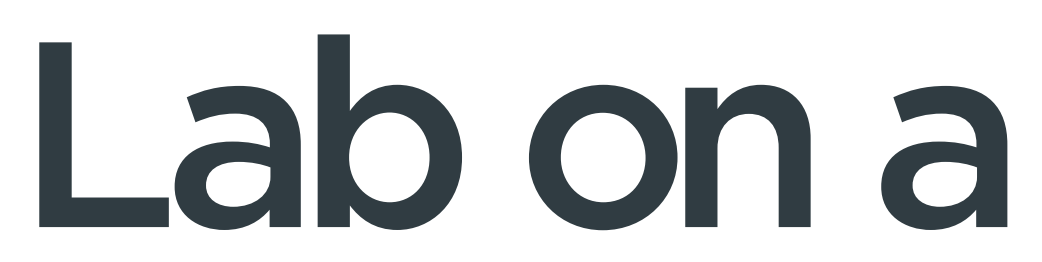

Devices and applications at the micro- and nanoscale rsc.li/loc

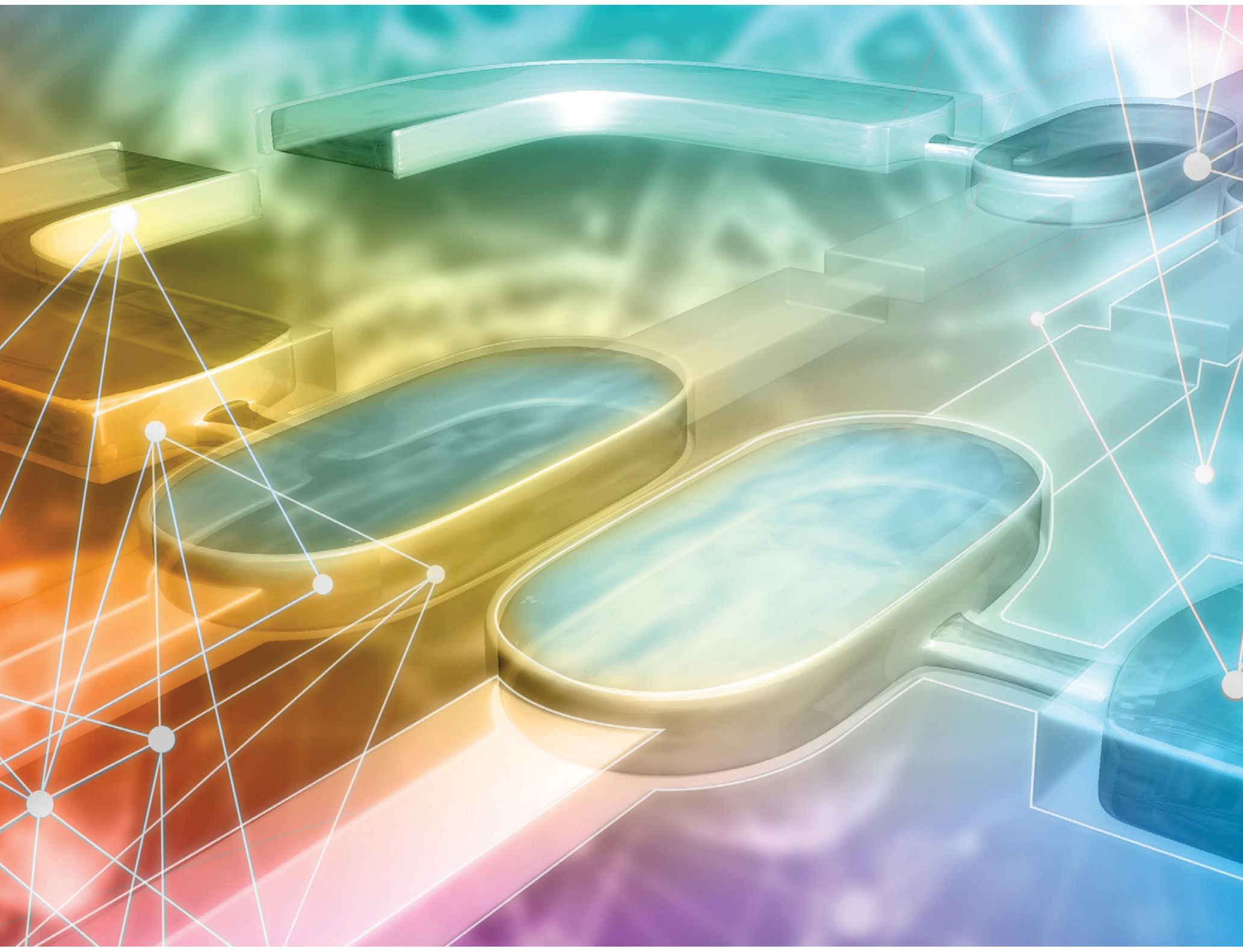

ISSN 1473-0197

\title{
PAPER
}

Volkert van Steijn et al.

Scalable microfluidic droplet on-demand generator for

non-steady operation of droplet-based assays 


\title{
Lab on a Chip
}

Check for updates

Cite this: Lab Chip, 2020, 20, 1398

\section{Scalable microfluidic droplet on-demand generator for non-steady operation of droplet- based assays $\dagger$}

\author{
Kartik Totlani, (D) a Jan-Willem Hurkmans, ${ }^{a}$ Walter M. van Gulik, ${ }^{b}$ \\ Michiel T. Kreutzer ${ }^{a}$ and Volkert van Steijn (ID*a
}

\begin{abstract}
We developed a microfluidic droplet on-demand (DoD) generator that enables the production of droplets with a volume solely governed by the geometry of the generator for a range of operating conditions. The prime reason to develop this novel type of DoD generator is that its robustness in operation enables scale out and operation under non-steady conditions, which are both essential features for the further advancement of droplet-based assays. We first detail the working principle of the DoD generator and study the sensitivity of the volume of the generated droplets with respect to the used fluids and control parameters. We next compare the performance of our DoD generator when scaled out to 8 parallel generators to the performance of a conventional DoD generator in which the droplet volume is not geometry-controlled, showing its superior performance. Further scale out to 64 parallel DoD generators shows that all generators produce droplets with a volume between $91 \%$ and $105 \%$ of the predesigned volume. We conclude the paper by presenting a simple droplet-based assay in which the DoD generator enables sequential supply of reagent droplets to a droplet stored in the device, illustrating its potential to be used in droplet-based assays for biochemical studies under non-steady operation conditions.
\end{abstract}

Received 7th November 2019, Accepted 21st March 2020

DOI: 10.1039/c9lc01103j

rsc.li/loc typically require sequential execution of a multiple of these operations. A common strategy to utilize these steady operations in a non-steady assay is to decouple them and execute them separately in a sequence, typically in separate dedicated devices, each one operated under steady flow conditions. $^{29}$ Although this decoupling strategy has been proven to be powerful for large screens, ${ }^{3,30}$ the number of operations possible during the screen is limited, often resorting to studying systems under batch conditions with all reagents present from the beginning, with end point measurements only. The application window of such dropletbased assays would hence significantly widen when developing an alternative strategy in which different operations can be integrated into the same device, operated in a non-continuous fashion. For example, a microdropletbased assay that would be very powerful is one that enables sequential addition of reagents to droplets containing cells and studying their behaviour over time under (semi) continuous conditions. However, the required intermittent addition of reagents to droplets stored in the device presents an important hurdle. In fact, integrating long-term culturing in a controlled environment with technology accessible to non-experts is seen as one of the great outstanding challenges in the field. ${ }^{31}$ While a few successful examples have been demonstrated to overcome the integration challenge using sophisticated devices with valves (integrated into the device 
or into the external connections to it), ${ }^{8,9,32-35}$ the complexity of these devices and their use prevents their widespread adoption. In this paper, we address this hurdle by presenting a scalable droplet on-demand approach that enables the sequential delivery of reagent droplets of a predefined volume to many locations in the assaying device at the same time using commercially available equipment.

Droplet on-demand (DoD) techniques in unbounded systems are well-established and find widespread use, for example, in the printing industry. One of the first DoD implementations in a microfluidic chip was provided by Attinger and co-workers. ${ }^{36}$ While most developments so far focused on single on-demand generators, ${ }^{37-40}$ several scaledout DoD approaches have been proposed. ${ }^{41,42}$ Guzowski et al., ${ }^{41}$ for example, developed a scaled-out device, generating droplets on demand in 16 parallel channels, using just one continuous phase inlet and one dispersed phase inlet, controlled by two external valves, with the volume of the droplets controlled by the duration the valves were opened. A foreseen challenge in adopting this approach in a more complicated device is that small differences in the hydrodynamic resistance across the device may lead to differences in the volume and timing of the generated droplets: a well-known problem in the scale out of multiphase microfluidic devices. ${ }^{43}$ Similar to making continuous droplet generation methods more robust in operation through geometric design, ${ }^{44-49}$ we present an innovative design that enables the generation of droplets

\section{(a)}
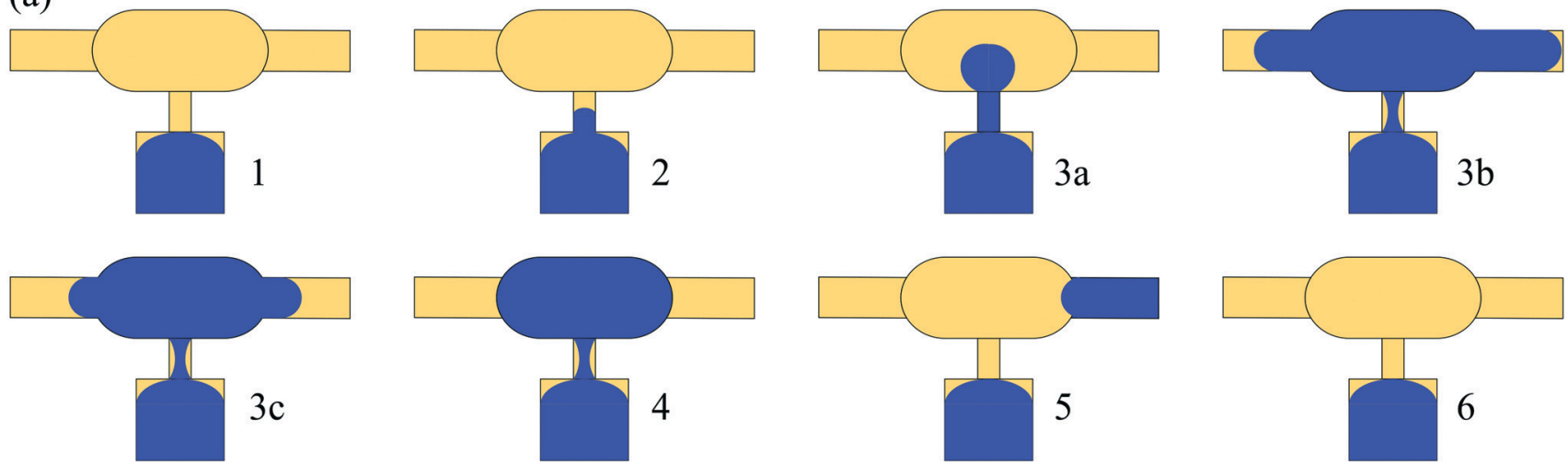

(b)
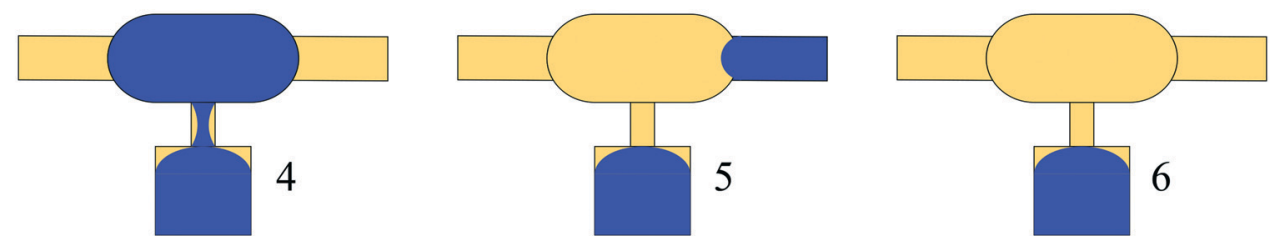

(c)
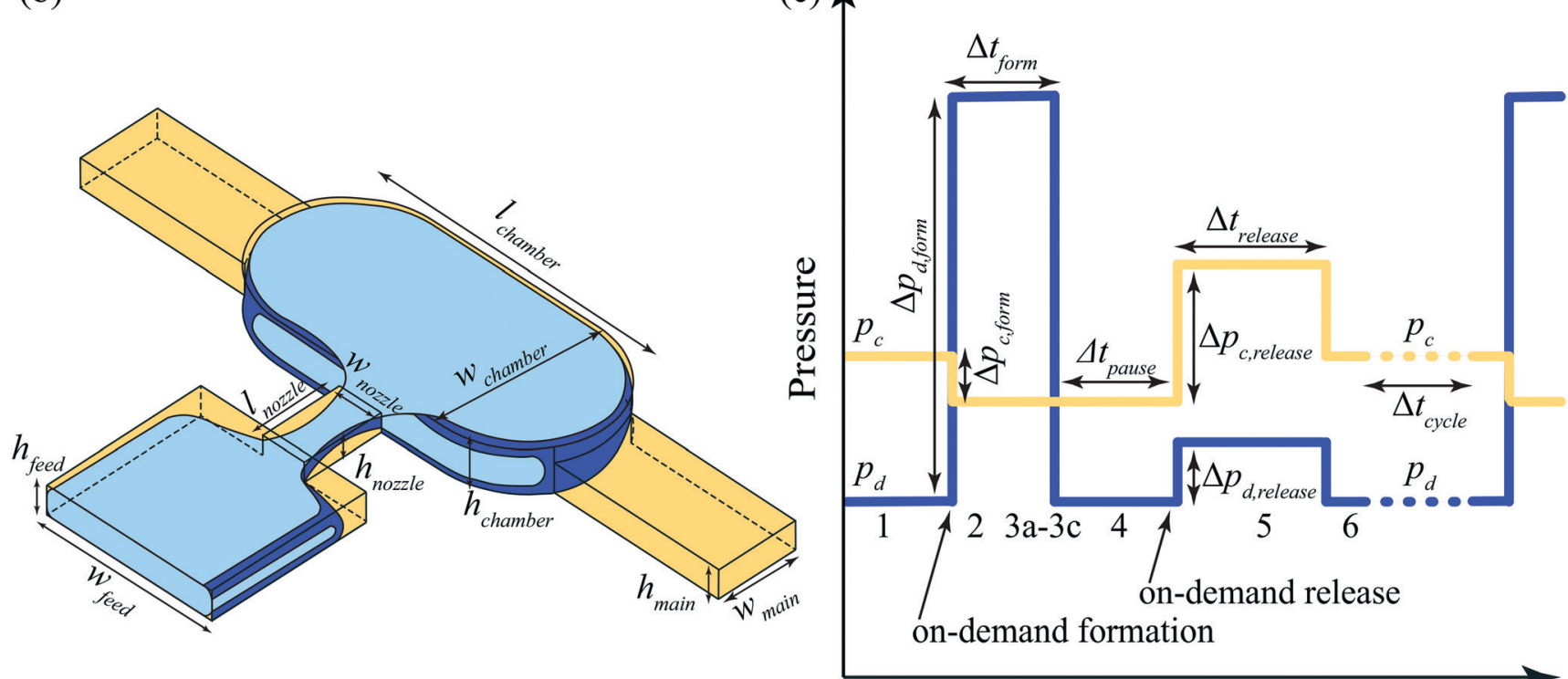

Time

Fig. 1 Droplet on-demand generator working principle and design. (a) Illustration of a DoD cycle, showing the dispersed phase steadily pressed against the nozzle (1), the dispersed phase filling the chamber (2-3c), the interfaces of the dispersed phase steadily pressed against the entrance of the main channel (4), and the release of a droplet with a volume similar to the volume of the chamber into the main channel (5), after which the new interface is steadily pressed against the nozzle (6), ready for a new DoD cycle. (b) Design of the DoD generator, illustrating the constrictions in width and height, between the feed channel of the dispersed phase and the chamber, and between the chamber and the main chamber. These two Laplace pressure barriers enable the decoupling of the formation of a droplet from its release, such that the generated droplet volume solely depends on the chamber volume for a range of operating conditions. (c) Pressure profiles used during a DoD cycle, showing that the formation and release of a droplet are initiated through two separate pressure pulses. 
with a volume solely governed by the geometry for a range of operating conditions. This insensitivity to local conditions enables robust scale out and the generation of droplets of the same volume at the same time at different locations in the device using an on-demand change in pressures in the fluid reservoirs from which the device is fed. In order to facilitate adoption of the here presented DoD approach within the community, we used commercially available equipment and microfluidic devices fabricated using routine protocols.

The working principle of our droplet on-demand generator is illustrated in Fig. 1(a), showing that the interface is steadily pressed against a nozzle (1) until, at will, the pressure in the fluid reservoir of the dispersed phase is temporarily elevated. This on-demand formation pulse pushes the interface through the nozzle (2) such that the dispersed phase starts filling the chamber (3a). From here, two modes of operation are possible, depending on the used control parameters: either the dispersed phase steadily fills the chamber until full or it initially spills into the main channel (3b) and subsequently retracts back into the chamber (3c). Importantly, both these modes result in a filled chamber, with the interfaces steadily pressed against the entrance of the main channel (4). By elevating the pressure in the fluid reservoir of the continuous phase, the dispersed phase is pushed from the chamber into the main channel. This ondemand release pulse leads to pinch-off and the release of a droplet (5) with a volume similar to the volume of the chamber. After release, the pressures are adjusted to their initial values, with the new interface being steadily pressed against the nozzle (6), ready for the next DoD cycle. Two innovative aspects of the here introduced two-step formation and release DoD approach are (1) the constrained growth of the droplet to a geometry-controlled (chamber) volume and (2) the decoupling between formation and release. While the use of chambers ${ }^{46,50}$ and the decoupling of formation and release based on flow modulation ${ }^{41,51-53}$ have been demonstrated before, it is the here introduced combination that makes the on-demand generation insensitive to the pressure upstream and downstream of the generator, enabling the robust scale out.

We start this paper by presenting a proof-of-principle for single droplet on-demand generation, showing the robustness of the approach, with regard to the operating conditions and used fluids. We next demonstrate the scalability of this approach by implementing the DoD design in 8 parallel channels, illustrating the importance of the chamber by comparing parallel DoD devices with and without chambers. Next, we demonstrate further scale out to 64 parallel channels, fed from just two fluid reservoirs, one for the continuous phase and one for the dispersed phase, through the use of a single (commercially available) pressure pump. We conclude this paper with an outlook on the use of the DoD generator in droplet-based assays, by demonstrating sequential feeding of reagent droplets to a droplet stored on a chip.

\section{Materials and methods}

\section{Device design}

The three-dimensional design of the microfluidic DoD generator is shown in Fig. 1(b), with two key features being the nozzle and the chamber. Firstly, the constriction between the feed and the chamber (i.e. nozzle) enables one to (1) steadily press the interface against the nozzle and initiate formation when raising the pressure above the Laplace pressure associated with the difference in height and width between the feed and nozzle. Secondly, the constriction between the chamber and main channel (2) facilitates confinement of the growth of the dispersed phase to the chamber and its subsequent release from the chamber into the main channel, again by changing the pressure in accordance with the Laplace pressure. Since these two Laplace pressure barriers act as valves, ${ }^{54}$ allowing the interface to only pass when the driving pressure (temporarily) exceeds the (static) Laplace pressure associated with the barriers, we hypothesize that the volume of the dispersed phase in the chamber after the formation pulse does not depend on the actual values of the strength and duration of the pulse. Even when the dispersed phase initially spills into the main channel and halts within a few channel widths from the chamber, it is expected to retract back into the chamber. This is again understood by considering the static Laplace pressure: the curvature of the interface in the main channel is higher than that in the chamber, such that the disperse phase flows back. Similarly, we do not expect the volume of the droplet after the release pulse to depend on the exact settings of the pulse. In order to prevent the unwanted formation or release of droplets due to pressure fluctuations present in the system, we constricted the channels not just in width, but also in height. The chamber, nozzle and main channel therefore have different heights, as shown in Fig. 1(b).

All DoD junctions presented in this paper have the same dimensions. The main channel is $50 \mu \mathrm{m}$ wide $\left(w_{\text {main }}\right)$ and 35 $\mu \mathrm{m}$ high $\left(h_{\text {main }}\right)$, the nozzle is $25 \mu \mathrm{m}$ wide ( $\left.w_{\text {nozzle }}\right), 25 \mu \mathrm{m}$ high $\left(h_{\text {nozzle }}\right)$ and $50 \mu \mathrm{m}$ long $\left(l_{\text {nozzle }}\right)$, and the feed of the dispersed phase is $100 \mu \mathrm{m}$ wide ( $w_{\text {feed }}$ ) and $35 \mu \mathrm{m}$ high $\left(h_{\text {feed }}\right)$. Of key importance are the dimensions of the chamber, because they impose the volume of the generated droplets. The chamber is $100 \mu \mathrm{m}$ wide ( $\left.w_{\text {chamber }}\right), 40 \mu \mathrm{m}$ high ( $\left.h_{\text {chamber }}\right)$, and $200 \mu \mathrm{m}$ long $\left(l_{\text {chamber }}\right)$, such that its volume is about $V_{\mathrm{ch}}=0.71 \mathrm{~nL}$. The chosen dimensions were based on initial tests performed in devices with different nozzle and chamber dimensions, with the ones with highly constricted nozzles and large chambers leading to premature droplet pinch-off, i.e. before the chamber was completely filled. The full design of the here used DoD generator is made available as an AutoCAD file in the ESI. $\dagger$

\section{Device operation}

A pressure flow controller (MFCS-4C 1000 mbar, Fluigent) was used to control the injection of the working fluids into 
the chips. The ports on this controller were connected to reservoirs containing the fluids using silicone tubing. PTFE tubing ( $0.5 \mathrm{~mm}$ ID, 1/16 inch OD, $60 \mathrm{~cm}$ in length, Kinesis) was used to connect the reservoirs to the tubing glued to the inlets of the chips with the use of PEEK connectors. Care was taken to use the same height difference between the outlet of the chips and the liquid levels in the reservoirs in order to have comparable contributions of the hydrostatic pressure between different sets of experiments. Relatively large reservoirs $(15 \mathrm{ml}$ Eppendorf tubes) were used to ensure a negligible change in liquid level over the course of an experiment.

The pressure pump was controlled using a custom made MATLAB script, which is provided in the ESI. $\dagger$ In each set of experiments, we ran the script to carry out 50 DoD cycles with a predefined pressure profile in an automated fashion. This profile is characterised by $p_{\mathrm{d}}, p_{\mathrm{c}}, \Delta p_{\mathrm{d} \text {,form }}, \Delta p_{\mathrm{c} \text {,form }}, \Delta t_{\text {form }}$, $\Delta t_{\text {pause }}, \Delta p_{\mathrm{d} \text {,release }}, \Delta p_{\mathrm{c} \text {,release }}, \Delta t_{\text {release }}$, and $\Delta t_{\text {cycle }}$, as defined in Fig. 1(c). While the main working principle has been explained before, with an increase in the dispersed phase pressure $\left(\Delta p_{\mathrm{d} \text {,form }}\right)$ initiating formation and an increase in the continuous phase pressure $\left(\Delta p_{\mathrm{c} \text {,release }}\right)$ initiating release, we did not yet explain the rationale behind the base line pressures $p_{\mathrm{d}}$ and $p_{\mathrm{c}}$ and the jumps $\Delta p_{\mathrm{c} \text {,form }}$ and $\Delta p_{\mathrm{d} \text {,release. }}$ The base line pressure $p_{\mathrm{c}}$ is chosen in order to obtain a desired (background) flow rate of the continuous phase through the main channel when the device is running steadily between droplet generation events. As the chosen value of $p_{\mathrm{c}}$ determines the local pressure in the chamber, the baseline pressure $p_{\mathrm{d}}$ is adjusted in accordance. In experiments, $p_{\mathrm{d}}$ was tuned manually in such a way that it resulted in the interface being steadily pressed against the nozzle. While the choice of $p_{\mathrm{c}}$ is not stringent, $p_{\mathrm{d}}$ should be chosen such that the interface neither gets pushed back into the feed channel nor through the nozzle. In the experiments performed, this was relatively straightforward. To ensure that the continuous phase does not prematurely break the neck of the dispersed phase by pushing it forward into the chamber after the formation pulse, we reduced the background flow of the continuous phase using $\Delta p_{\text {c,form }}$ when initiating formation. When initiating release, an extra pressure of $\Delta p_{\mathrm{d} \text {,release }}$ is added to the dispersed phase in order to avoid the dispersed phase being pushed back into the feed channel. With this in mind, we noticed in the initial experiments that the window of operation is relatively large for the release stage. In the experiments, we hence focus on the sensitivity of the droplet volume with respect to the operating parameters associated with the formation stage.

\section{Device fabrication}

The microfluidic devices were fabricated using standard soft lithographic methods. ${ }^{55}$ To obtain channels with three different heights in the same device, we spun three layers of the negative photoresist SU-8 (micro resist technology $\mathrm{GmbH}$ ) on top of each other on a 4 inch silicon wafer and exposed each layer to near UV (EVG 620, EV Group) through a separate mask. These masks were designed using AutoCAD 2015 (Autodesk) and printed on transparencies using a high resolution printer (CAD/Art (Oregon, USA)). The first mask featured all channels of the microfluidic device, while the channel connecting the feed of the dispersed phase to the chamber (i.e. the nozzle) was not included on the second mask in order to make a nozzle with a constriction in height. The third mask only contained the chamber in order to make it higher than all the other channels. The designs of the three masks are made available in the ESI. $\dagger$ Unless stated otherwise, microfluidic devices were constructed from a 25 $\mu \mathrm{m}$ thick first layer (SU-83025), a $10 \mu \mathrm{m}$ thick second layer (SU-83005), and a $5 \mu \mathrm{m}$ thick third layer (SU-83005). After spin-coating a layer, it was soft baked, exposed, and post baked, following the guidelines provided by the manufacturer. The resulting wafer was then developed with mr-Dev 600 (micro resist technology $\mathrm{GmbH}$ ), washed with isopropyl alcohol, spin dried, hard baked at $150{ }^{\circ} \mathrm{C}$ for 30 minutes, and slowly cooled down on a hot plate to avoid cracks in the SU-8 structures. Before using the wafer as a master for replica moulding in PDMS, its surface was silanized by exposing it to vapours of $1 \mathrm{H}, 1 \mathrm{H}, 2 \mathrm{H}, 2 \mathrm{H}^{-}$ perfluorooctyltrichlorosilane in a depressurized desiccator.

PDMS devices were made by mixing $80 \mathrm{~g}$ of PDMS elastomer and $8 \mathrm{~g}$ of curing agent (Dow Corning, Sylgard 184 elastomer kit), degassing the mixture and pouring it over the master placed in a 5 inch Petri dish. The PDMS mixture was cured at $70{ }^{\circ} \mathrm{C}$ for 3 hours in an oven, gently removed from the wafer, and cut to size. The inlets and outlets were punched with a $1.5 \mathrm{~mm}$ biopsy punch and the resulting PDMS moulds were washed with ethanol to remove dust and debris. The PDMS moulds and PDMS spin coated glass slides $(25 \mathrm{~mm} \times 75 \mathrm{~mm})$ were exposed to an oxygen plasma (Harrick, PDC-002) for $140 \mathrm{~s}$ at a pressure of 0.2-0.4 mbar and subsequently pressed together to bond them. The obtained microfluidic devices were then baked at $140{ }^{\circ} \mathrm{C}$ for at least 4 hours to regain the hydrophobicity of PDMS. Small pieces of PEEK tubing (0.02 inch ID, 1/16 inch OD, $2 \mathrm{~cm}$ in length, IDEX Health and Science) were inserted into the inlets and glued tight.

\section{Working fluids}

Unless stated otherwise, experiments were done with demi water as the dispersed phase and HFE-7500 (Novec, $3 \mathrm{M}$ ) as the continuous phase, with $0.1 \mathrm{v} / \mathrm{v} \%$ Picosurf-1 (Sphere Fluidics) dissolved in HFE-7500 to stabilise the interfaces and ensure full wetting of the walls by the continuous phase. The fluid properties for this system at room temperature are: 1 $\mathrm{mPa} \mathrm{s}$ and $1.24 \mathrm{mPa}$ s for the viscosities of the dispersed and continuous phases, respectively, and $2.4 \mathrm{mN} \mathrm{m}^{-1}$ for the interfacial tension.

\section{Image acquisition and analysis}

For each set of experiments, 50 DoD cycles were captured using a TIS camera (DMK 33UJ003, The Imaging Source) 
mounted on an internally illuminated microscope (Axiovert S100, Zeiss). Images were captured through a combination of a $5 \times$ objective and a $0.63 \times$ mount objective. Images were acquired at a resolution of $3856 \mathrm{px} \times 500 \mathrm{px}$ and at a frame rate of $1 \mathrm{fps}$. To avoid the unnecessary acquisition of images in experiments with long cycle times, a custom-made MATLAB script was used to only acquire images during the DoD cycles, by sending a trigger signal to the camera right before the signal to initiate the formation pulse was sent to the pressure pump. In addition, a high speed camera (Phantom V9, Vision Research) was used to capture the dynamics of the DoD formation process.

Images were processed to determine the length, $L$, of the droplets after they were fully released into the main channel. The length was subsequently used to determine the volume using $V=\left[h_{\text {main }} w_{\text {main }}-(4-\pi)\left(2 / h_{\text {main }}+2 / w_{\text {main }}\right)^{-2}\right]\left(L-w_{\text {main }} /\right.$ 3) established in earlier work. ${ }^{56}$ For each set of 50 DoD cycles, we calculated the average and standard deviation of the volume. We used the coefficient of variation (CoV), defined as the ratio between the standard deviation and the average, as a measure of the monodispersity.

\section{Results and discussion}

\section{Single droplet on-demand: robustness in operation}

In order to test the robustness in operation of the ondemand droplet generator, we conducted experiments in which we varied the main control parameters. We start by detailing how the strength of the pressure pulse $\left(\Delta p_{\mathrm{d}, \text { form }}\right)$ and the duration of the pressure pulse $\left(\Delta t_{\text {form }}\right)$ affect the sensitivity of the volume of the generated droplets and their monodispersity.

The effect of pulse strength is first detailed for the dynamics of the DoD process, before showing its influence on the resulting droplet volume. Fig. 2(a) illustrates the dynamics for different pulse strengths as a series of three microscopy images, one during filling, one before release from the chamber, and one after release into the main channel. For the lower pulse strengths of 15 and 20 mbar, the dispersed phase fills the chamber until it is full, after which the dispersed phase steadily resides inside the chamber, until it is released. For larger pulse strengths, we observe that the dispersed phase initially overflows the chamber, spilling into the main channel, with larger pulse strengths leading to larger overshoots as evident from the left column in Fig. 2(a). After this overshoot, the interfaces halt within a few channel widths from the chamber. At that instant, the dispersed phase pressure in the main channel is higher than that in the chamber due to the curvature of the interfaces imposed by the main channel. As a result, the dispersed phase flows back into the chamber. Despite the overshoot for larger pulse strengths and the difference in dynamics for low and high pulse strengths, the formation pulse results in the same steady outcome: a chamber filled with the dispersed phase, from which a droplet can be released upon a second pulse.
For pulse strengths between 15 to 35 mbar, we find that the generated droplets have a volume $(V)$ similar to the volume of the chamber $\left(V_{\mathrm{ch}}\right)$, as shown in Fig. 2(b), with all the corresponding $\mathrm{CoV}$ values below 0.03. Pulse strengths below 15 mbar were not sufficiently powerful to push the dispersed phase through the nozzle. This is in line with the order of magnitude of the (over)pressure required to push the interface quasi-statically through the nozzle, which is about 4 mbar as estimated from the Laplace law, $\gamma\left(2 / w_{\text {nozzle }}+\right.$ $\left.2 / h_{\text {nozzle }}\right)$. Although not shown, the droplet formation process works in a similar fashion for pulse strengths of 40 and 45 mbar and the device operation was insensitive to pulse strengths up to $45 \mathrm{mbar}$. For larger pulse strengths (at $\Delta t_{\text {form }}$ $=0.2 \mathrm{~s}$ ), we observed that the interface penetrated all the way to the exit of the downstream channel such that retraction back into the chamber did not occur.

The effect of the duration of the pulse $\left(\Delta t_{\text {form }}\right)$ on the dynamics of DoD generation is illustrated in Fig. 2(c). The behaviour is very similar to that observed for different pulse strengths; the chamber is gradually filled with the dispersed phase for short pulses, while an initial overshoot and subsequent retraction occur for longer pulses. The resulting droplet volume is insensitive to the applied pulse duration for the here reported range of duration times, see Fig. 2(d), with the corresponding $\mathrm{CoV}$ values below 0.03. Outside the reported range, the interface was not pushed through the nozzle for shorter pulses, while it overshot to the exit for longer pulses. We note that the droplet volumes in Fig. 2(d) are systematically lower than the chamber volume. With the pre-formed droplets in the chamber similar in volume in Fig. 2(a) and (c), we expect that the lower value is caused by partial backflow of the dispersed phase into the nozzle before complete pinch-off during release.

We conclude the experiments on pulse strength and pulse duration by returning to our original hypothesis that the volume of the dispersed phase in the chamber after the formation pulse and the volume of the generated droplet after the release pulse have a low sensitivity with respect to the actual values of the pressure pulses used, as long as the driving pressure is raised above the static Laplace pressure associated with the barriers for a sufficient amount of time. The data presented in Fig. 2 confirms that (i) droplets are not generated for driving pressures below the static Laplace pressure and (ii) droplets are generated for higher pressures, with the performance of the generator, characterized in terms of droplet volume and variations therein, having a low sensitivity to the actual settings of the pressure pulse, for pulse strengths and pulse duration times in the range between 15 and 45 mbar and 0.3 and 0.7 seconds. This low sensitivity enables different DoD generators in a scaled-out device to produce similarly sized droplets, even when the local pressures near the different DoD generators are different.

To further substantiate the robustness in operation of the DoD generator, we studied the sensitivity of the volume of the generated droplets with respect to the time between two 
(a)

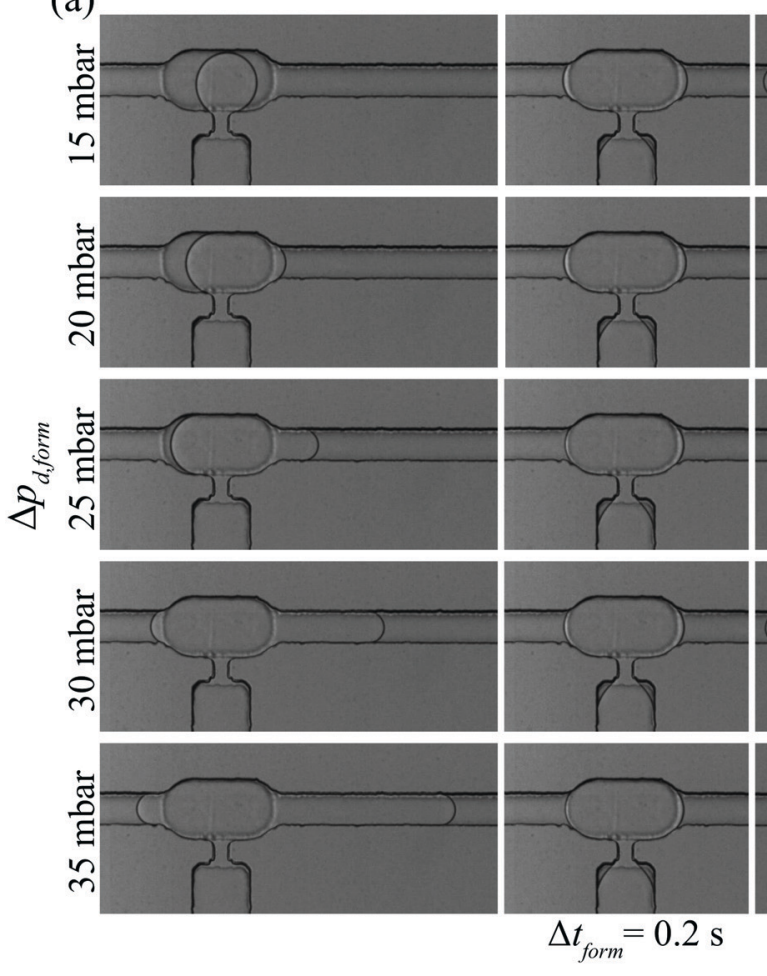

(c)

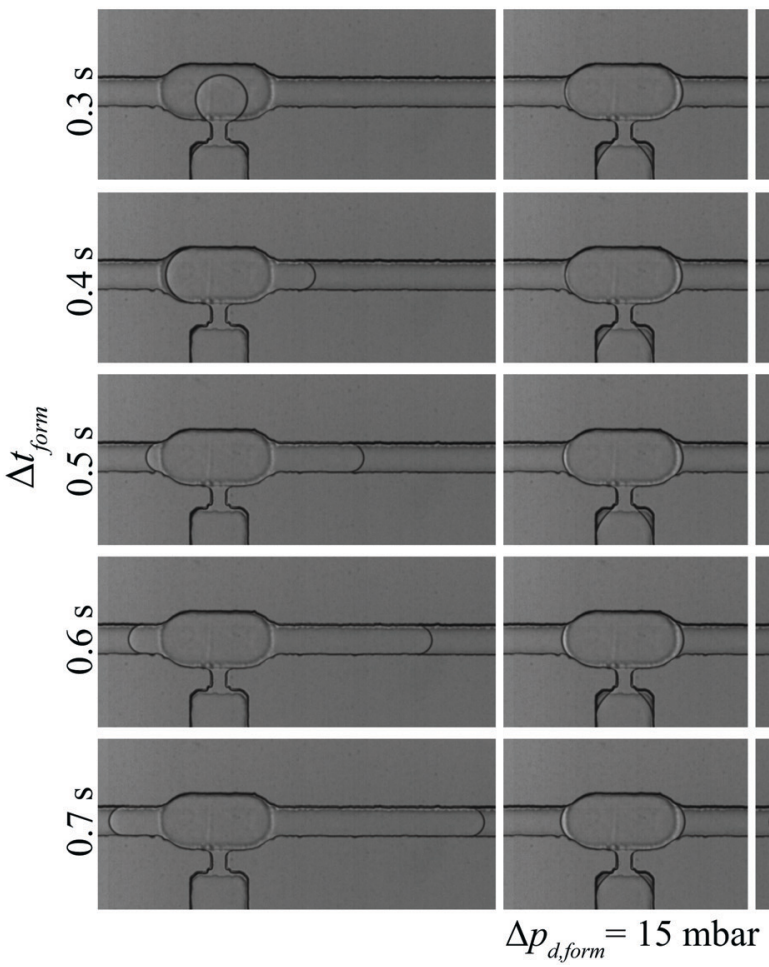

(b)
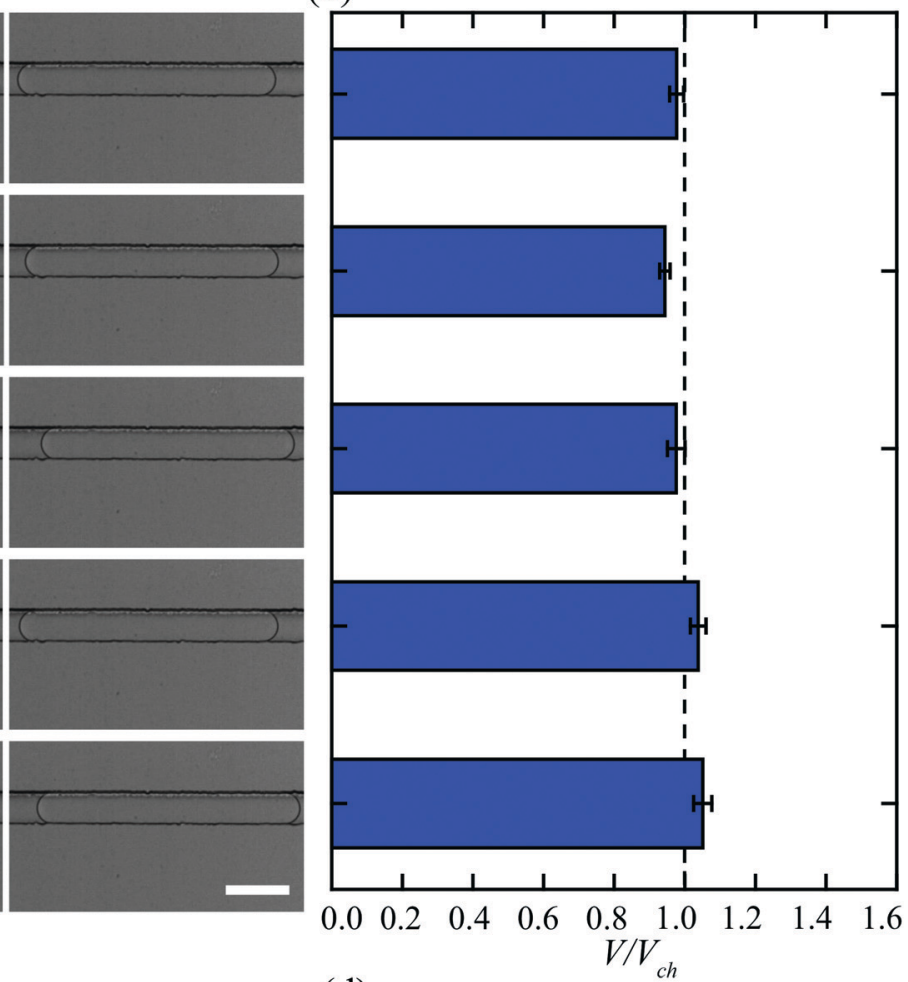

(d)
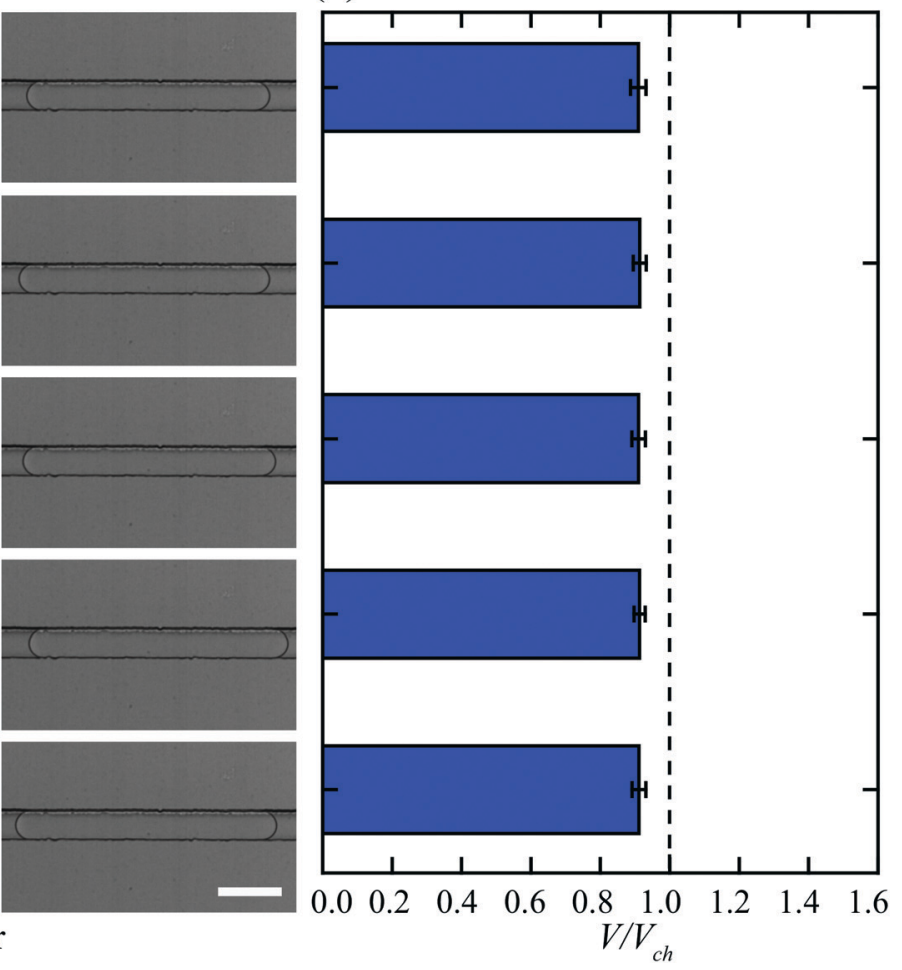

Fig. 2 Characterization of the DoD generator, showing its robustness in operation with respect to the two main control parameters. (a) Effect of pulse strength $\left(\Delta p_{\mathrm{d}, \text { form }}\right)$ on the dynamics of droplet generation, illustrated using micrographs taken during filling (left), before release (middle), and after release (right). See the ESI $\dagger$ for the corresponding movie. (b) Resulting normalized droplet volume for 50 DoD cycles, with the error bars representing the standard deviation. (c) Effect of pulse duration ( $\Delta t_{\text {form }}$ ) on the dynamics of droplet generation (see the ESI $\dagger$ for the corresponding movie) with the resulting normalized volumes in (d). Pressure profile: $p_{\mathrm{d}}=22 \mathrm{mbar}, p_{\mathrm{c}}=30 \mathrm{mbar}$, and $\Delta p_{\mathrm{c} \text {,form }}=3 \mathrm{mbar}$ for $\Delta p_{\mathrm{d} \text {,form }}$ variation and $p_{\mathrm{d}}=26 \mathrm{mbar}, p_{\mathrm{c}}=36 \mathrm{mbar}$, and $\Delta p_{\mathrm{c} \text {,form }}=2.8 \mathrm{mbar}$ for $\Delta t_{\text {form }}$ variation. The other parameters that define the pressure profile were kept constant at $\Delta t_{\text {pause }}=15 \mathrm{~s}, \Delta p_{\mathrm{d} \text {,release }}=5 \mathrm{mbar}, \Delta p_{\mathrm{c} \text {,release }}=12 \mathrm{mbar}, \Delta t_{\text {release }}=5 \mathrm{~s}$, and $\Delta t_{\text {cycle }}=8 \mathrm{~s}$. Scale bars in (a) and (c): $100 \mu \mathrm{m}$. 


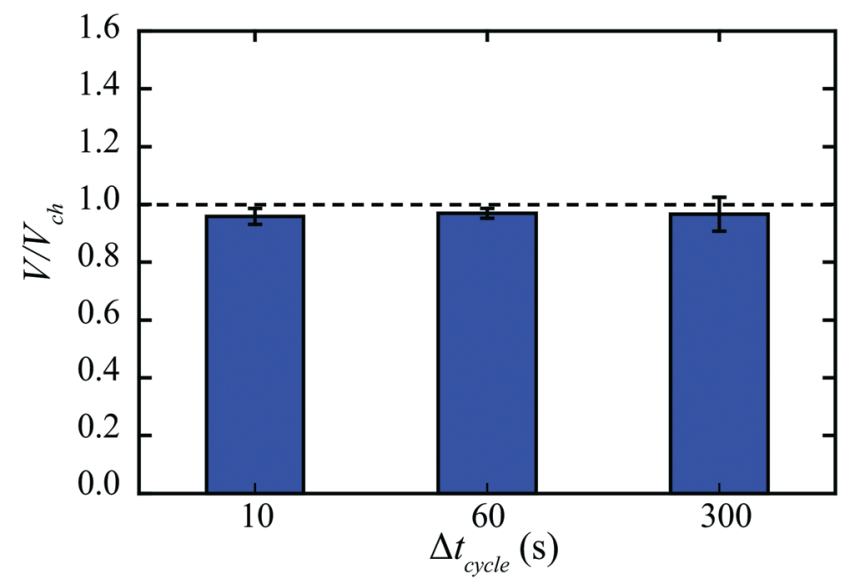

Fig. 3 Effect of cycle time ( $\Delta t_{\text {cycle }}$ ) on the generated droplet volume and monodispersity. Pressure profile: $p_{\mathrm{d}}=26 \mathrm{mbar}, p_{\mathrm{c}}=52 \mathrm{mbar}$, $\Delta p_{\mathrm{d}, \text { form }}=20 \mathrm{mbar}, \Delta p_{\mathrm{c}, \text { form }}=3 \mathrm{mbar}, \Delta t_{\text {form }}=0.2 \mathrm{~s}, \Delta t_{\text {pause }}=30 \mathrm{~s}$, $\Delta p_{\mathrm{d}, \text { release }}=5 \mathrm{mbar}, \Delta p_{\mathrm{c}, \text { release }}=12 \mathrm{mbar}, \Delta t_{\text {release }}=5 \mathrm{~s}$.

droplet formation cycles $\left(\Delta t_{\text {cycle }}\right)$. The volume of the generated droplets is insensitive for the here studied range as shown in Fig. 3. The CoV for $\Delta t_{\text {cycle }}=10 \mathrm{~s}$ and $60 \mathrm{~s}$ is 0.02 , while it is 0.06 for $\Delta t_{\text {cycle }}=300 \mathrm{~s}$. In this set, we observed slight differences in the location of the interfaces when pressed against the entrance of the main channel in different DoD cycles, which may be caused by (long term) variations in the base line pressures.

Before further characterizing the performance of the DoD generator, we comment on the feeding frequencies (or throughput) that can be achieved in the context of its intended use. One of the foreseen areas of its application is in bioprocess engineering, where it can be used for long term cultivation of cells under sequential-batch/fed-batch/semicontinuous conditions, with control over the produced metabolites through the controlled supply of nutrients. The required feeding frequency then depends on the desired growth rate of the cells. The simplest estimate of the order of magnitude of the required feeding frequency is obtained by considering a chemostat, for which the required volumetric flow rate of nutrients $(F)$ solely depends on the volume in which the cells are cultured $\left(V_{\mathrm{r}}\right)$ and the desired cell growth rate $\left(\mu_{\text {cell }}\right)$ as $F=V_{\mathrm{r}} \mu_{\text {cell }}$. For feeding nutrient droplets of volume $V$ at a frequency $f$, the required frequency simply is $f$ $=\left(V_{\mathrm{r}} / V\right) \mu_{\text {cell }}$. For typical nutrient-controlled growth rates of the order of $0.1 \mathrm{~h}^{-1}$ and nutrient volumes of the order of 10-100 times the cell culture volume, the expected feeding frequency is of the order of 1-10 droplets per hour. This is well in the range of feeding frequencies possible with the DoD generator, with its maximum generation frequency primarily depending on the time it takes for the interface to retract back from the main channel into the chamber after the formation pulse. This typically occurs within 5-10 seconds, such that we used 15-30 seconds of pause time $\left(\Delta t_{\text {pause }}\right)$ in the experiments reported in Fig. 2 and 3 to ensure that the dispersed phase is steadily pressed against the entrance of the main channel before releasing it. Considering also the other duration times in a DoD cycle $\left(\Delta t_{\text {form }}<1 \mathrm{~s}, \Delta t_{\text {release }} \sim 5\right.$ $\mathrm{s}, \Delta t_{\text {cycle }} \sim 10-300 \mathrm{~s}$ ), the maximum generation frequency is about one droplet per minute.

\section{Single droplet on-demand: robustness in used fluids}

To test the versatility of the DoD generator in dispensing different types of aqueous fluids, we performed experiments in which we systematically changed the viscosity of the dispersed phase from $1 \mathrm{mPa} \mathrm{s}$ to $220 \mathrm{mPa} \mathrm{s}$ by adding glycerol (Sigma Aldrich) to demi water in different weight percentages (0-90\%). While we kept all control parameters the same except for the one varied in the measurement series in Fig. 2, we found that the strength $\left(\Delta p_{\mathrm{d}, \text { form }}\right)$ and duration $\left(\Delta t_{\text {form }}\right)$ of the formation pulse, as well as the equilibrium pressures $\left(p_{\mathrm{c}}, p_{\mathrm{d}}\right)$, needed adjustments for each viscosity. More specifically, a higher viscosity required a higher strength and/or duration of the pulse for it to be of sufficient power to push the interface through the nozzle. Additionally, higher viscosities did not show the overshoot, as observed for the experiment with demi water, increasing the window of operation. Although different settings were needed for different viscosities (see the ESI $\dagger$ ), it is important to stress that, as before, a range of operating conditions could easily be identified in which the droplet volume was insensitive to the operating conditions. Thus, the generated droplets have a volume closely resembling the volume of the chamber, with CoV values below 0.02 , regardless of the dispersed phase viscosity, as shown in Fig. 4(a).

A key aspect in the design of droplet-based microfluidic devices is ensuring that droplets, during and after their formation, are not in direct contact with the microchannel walls, as the resulting contact lines and hysteresis in their motion generally makes operation of the device amendable for control. To ensure full wetting of the microchannel walls by the continuous phase in the so far reported experiments, we used $0.1 \mathrm{v} / \mathrm{v} \%$ Picosurf- 1 as a surfactant. To test the device operation under partial wetting conditions, we also performed one experiment without the surfactant. The static contact angle as measured by dispensing a droplet of pure HFE-7500 on an untreated PDMS surface submerged in demi water is $74^{\circ}$, while the interfacial tension between the fluids is about $48 \mathrm{mN} \mathrm{m}^{-1}$. Although the droplets were reasonably monodisperse (CoV below 0.06), the volume of the droplets was significantly smaller than the volume of the chamber, see Fig. 4(b). Fig. 4(c) illustrates the dynamics of the droplet release process under full wetting and partial wetting conditions. Before the release pulse, the chamber is completely filled with the dispersed phase in both cases. As soon as the release pulse is applied, we observe contact line pinning under partial wetting conditions, whereas the interface moves fluently under full wetting conditions. Hysteretic behaviour is in line with the higher required release pulse strength $\left(\Delta p_{\mathrm{c} \text {,release }}=25\right.$ mbar versus $\left.10 \mathrm{mbar}\right)$. Due to interface pinning near the exit of the chamber, the dispersed phase is partially pushed back into the nozzle 
(a)

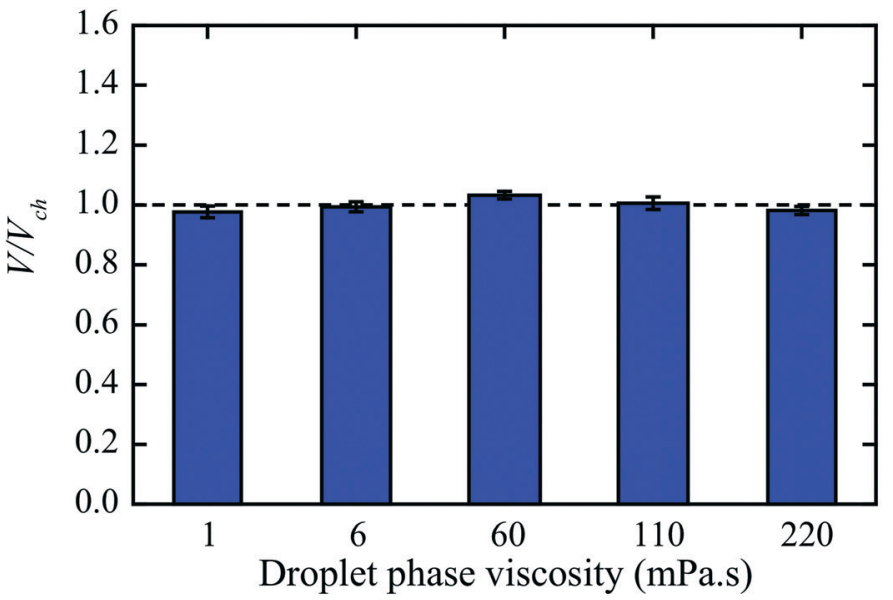

(b)

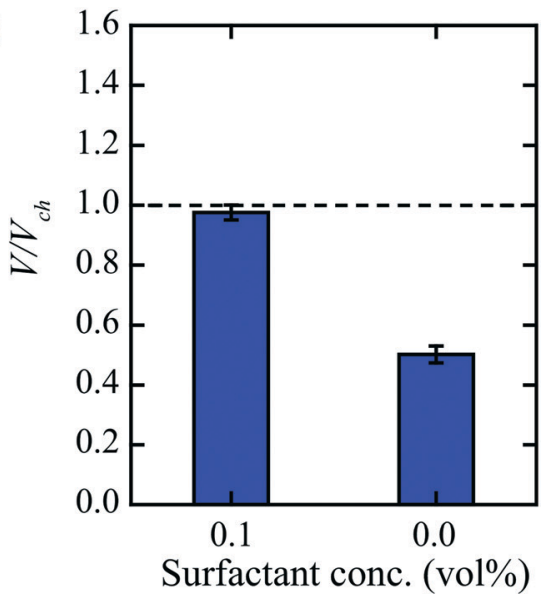

(c)
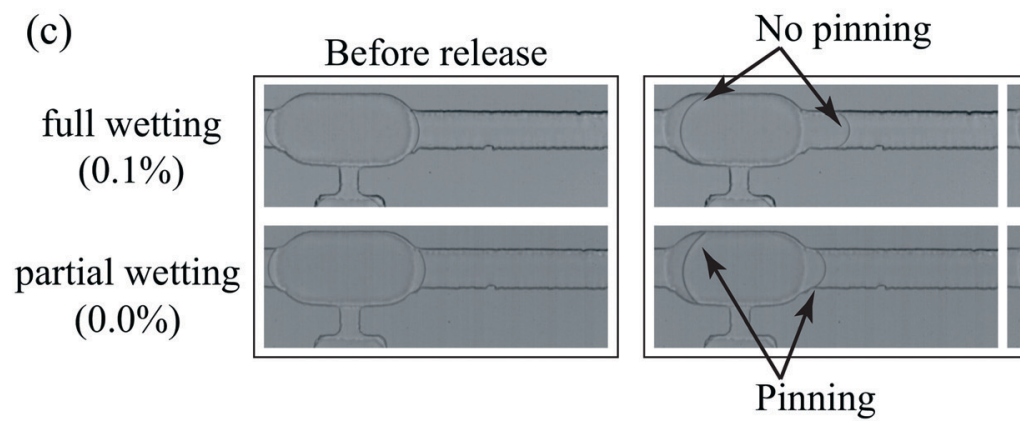

During release

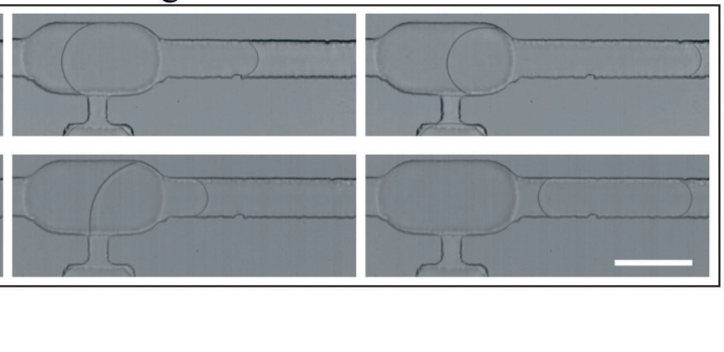

Fig. 4 Characterization of the DoD generator, showing its robustness in operation with respect to the use of different fluids. (a) Droplet volumes (average and standard deviation) for different viscosities of the dispersed phase. (b) Droplet volumes (pure demi water) generated with oil with and without a surfactant, showing the importance of ensuring full wetting conditions for the DoD generator to operate as intended. (c) Corresponding time series, showing that the dispersed phase is fluently pushed out of the chamber for the full wetting case, while contact line pinning causes the dispersed phase to be partially pushed back into the nozzle, resulting in droplets that are smaller than the chamber volume. See the ESI $\dagger$ for the corresponding movies. Used pressure profile for the full wetting case: $\Delta p_{\mathrm{d} \text {,form }}=40 \mathrm{mbar}, \Delta t_{\text {form }}=0.2 \mathrm{~s}$. for the rest of the settings, see the caption to Fig. 2. Used pressure profile for the partial wetting case: $p_{\mathrm{d}}=54 \mathrm{mbar}, p_{\mathrm{c}}=35 \mathrm{mbar}, \Delta p_{\mathrm{d} \text {,form }}=40 \mathrm{mbar}, \Delta p_{\mathrm{c} \text {,form }}=2 \mathrm{mbar}, \Delta t_{\mathrm{form}}=0.2$ $\mathrm{s}, \Delta t_{\text {pause }}=15 \mathrm{~s}, \Delta p_{\text {d,release }}=5 \mathrm{mbar}, \Delta p_{\mathrm{c}, \text { release }}=30 \mathrm{mbar}, \Delta t_{\text {release }}=5 \mathrm{~s}, \Delta t_{\text {cycle }}=8 \mathrm{~s}$. Scale bar: $100 \mu \mathrm{m}$.

before pinch-off occurs, resulting in droplets smaller than the chamber volume, see also the corresponding movie in the ESI.† This data set illustrates the importance of working under full wetting conditions for the intended operation of the DoD generator. In case it is not possible to choose the combination of working fluids such that the continuous phase fully wets the walls, one may be able to modify the roughness and chemical nature of the microchannel walls to achieve this.

\section{Scaling out droplet on-demand: the importance of} decoupling formation and release

After the thorough characterization of a single DoD generator, we demonstrate that its robustness in operation enables the scale out by presenting a device in which droplets are generated in 8 parallel generators, all fed from one fluid reservoir for the continuous phase and one for the dispersed phase through the use of a single pressure pump. To illustrate the importance of the here introduced decoupling strategy through inclusion of the chamber in the design, we also fabricated a device without chambers, see Fig. 5(a). This device was fabricated using the same fabrication protocol, with omission of the third layer. The obtained data for 50 DoD cycles shows that the volume of the droplets closely resembles the volume of the chamber for the $8 \mathrm{DoD}$ generators with chambers, with values of the monodispersity within 0.03 . By contrast, large variations in droplet size and monodispersity (CoV values up to 0.16) are seen for the device without chambers, see Fig. 5(b). These differences in droplet volume are attributed to the differences in volumetric flow rates arising from differences in the dimensions of the channels leading to the generators. This is well-known for the type of distributor used here, ${ }^{57}$ where the fluids are supplied to the droplet generators through a series of bifurcations of the inlet channels. An alternative to this treelike distributor is a so-called ladder-like distributor, ${ }^{49,58,59}$ which is designed to have a negligible pressure drop over the distribution channels in comparison to the pressure drop over the droplet generators. This is achieved by feeding the phases to the generators through relatively large inlet channels, which requires a three-dimensional network of connecting channels. This can be achieved by adding a fluid distribution layer on top of the chip and may reduce the 
(a)
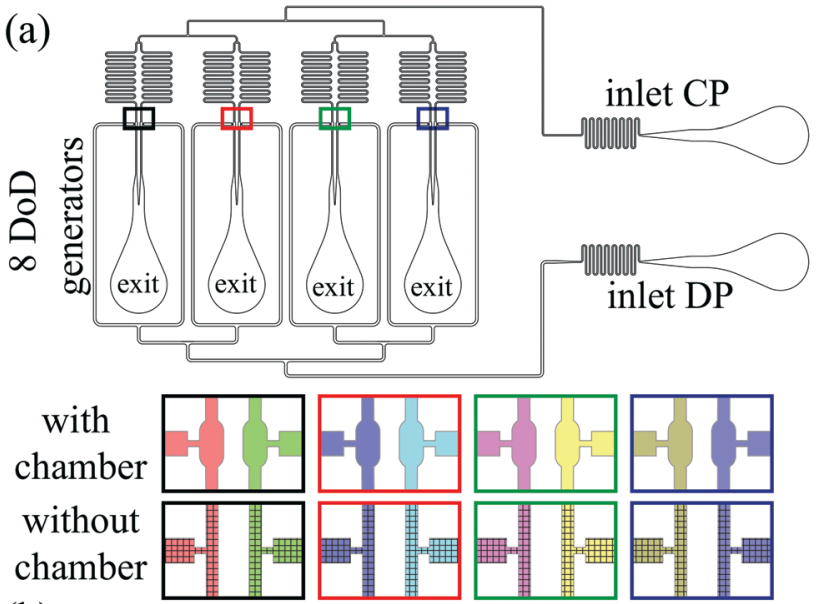

(b)

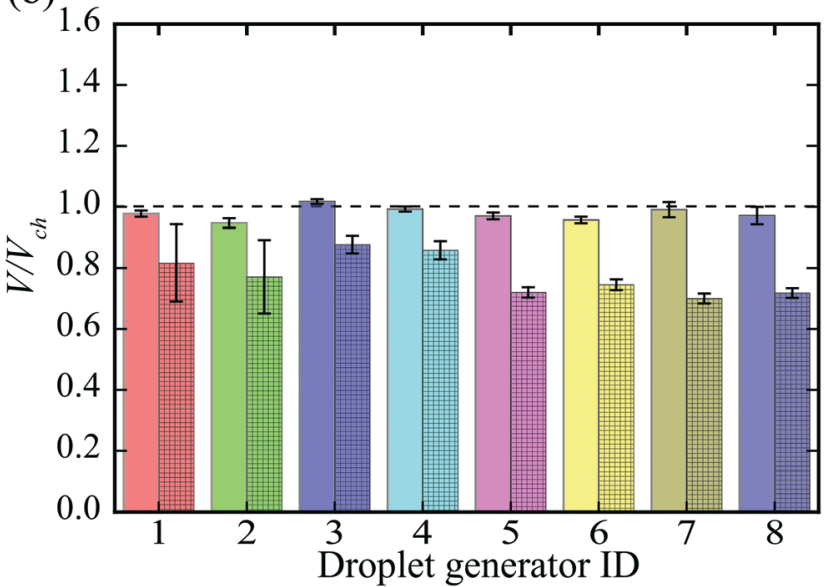

Fig. 5 Scale out of the DoD generator. (a) Design of the device with 8 parallel DoD generators, all fed from one fluid reservoir for the continuous phase and one for the dispersed phase through the use of a single pressure pump. (b) Average droplet volume in 50 DoD cycles and corresponding monodispersity (error bars), for a device with chambers (unhatched) and without chambers (hatched), demonstrating the importance of the chamber in the design. Used pressure profile for the device with chambers: $p_{\mathrm{d}}=28 \mathrm{mbar}, p_{\mathrm{c}}=38$ mbar, $\Delta p_{\text {d,form }}=45 \mathrm{mbar}, \Delta p_{\mathrm{c}, \text { form }}=3.7 \mathrm{mbar}, \Delta t_{\text {form }}=0.4 \mathrm{~s}, \Delta t_{\text {pause }}=$ $30 \mathrm{~s}, \Delta p_{\text {d, release }}=0 \mathrm{mbar}, \Delta p_{\mathrm{c}, \text { releas }}=180 \mathrm{mbar}, \Delta t_{\text {release }}=10 \mathrm{~s}, \Delta t_{\text {cycle }}=$ $8 \mathrm{~s}$. Used pressure profile for the device without chambers: the same, except for $p_{\mathrm{d}}=30 \mathrm{mbar}$ and $p_{\mathrm{c}}=39 \mathrm{mbar}$.

variations in droplet volume observed in the device without chambers, because variations in the volumetric flows to the different generators arising from fabrication tolerances may be smaller in ladder-like distributors than in tree-like distributors. Importantly, the here presented DoD generator (with chambers) can be parallelized using a tree- or ladderlike flow distributor, because the DoD generators produce droplets with a volume similar to the chamber volume, even when the pressures and volumetric flows differ between the parallel DoD generators.

We envision this DoD approach to be used as a reagent/ nutrient delivery tool in droplet-based assays for long-term experiments where tens to hundreds of experiments can be performed in parallel. To demonstrate the ability to further (a)

Before formation pulse

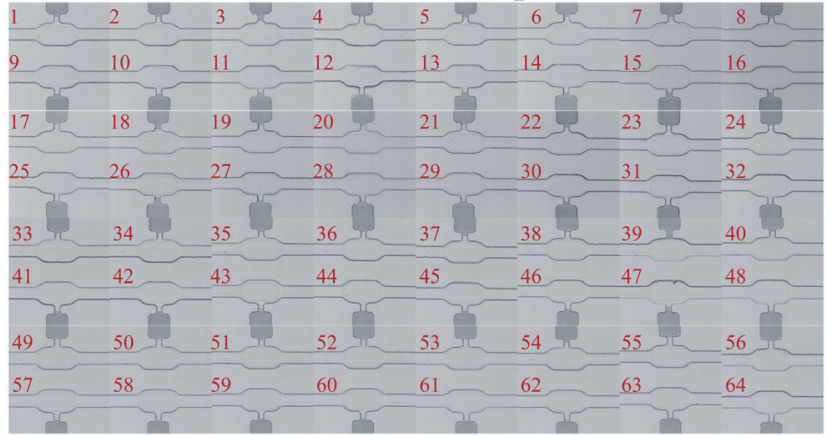

(b)

Filling

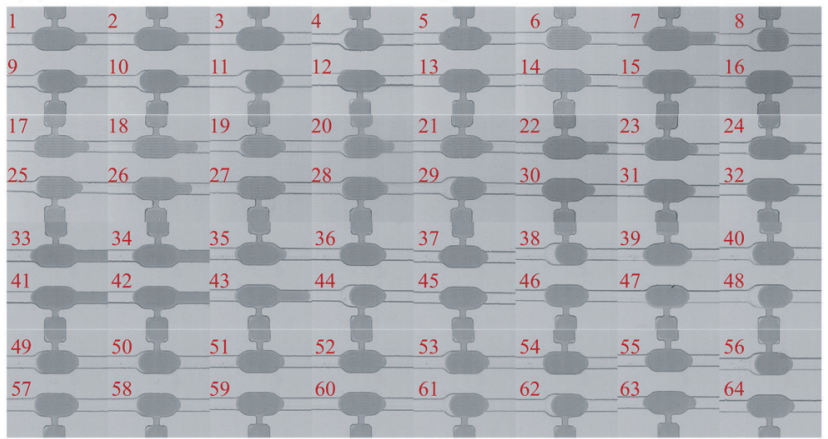

(c)

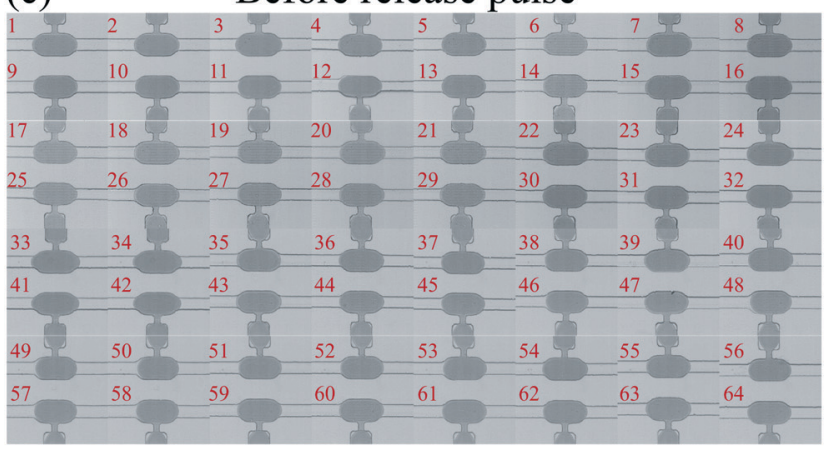

(d)

Releasing

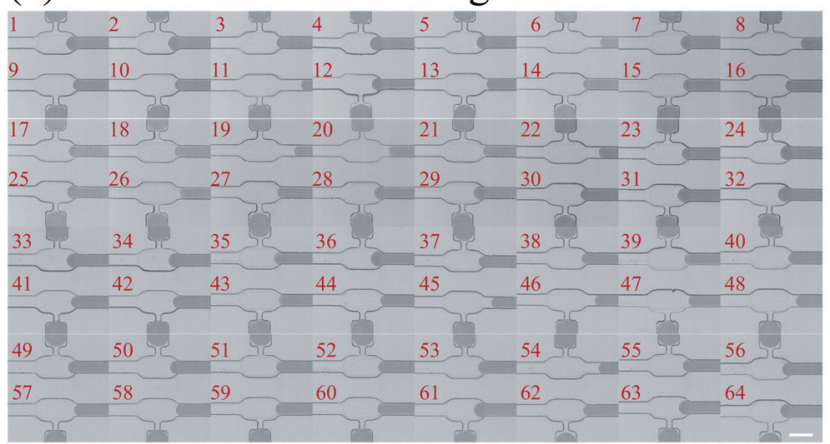

Fig. 6 Scale out of the DoD generator to 64 parallel DoD generators on a single chip. Operation of the device is characterized using four microscopic snapshots taken: Before applying the first pressure pulse that initiates the formation of droplets (a), immediately after the formation pulse (b), before applying the second pressure pulse that initiates the release of droplets (c), and immediately after the release pulse (d). Methylene blue was added to demi water to enhance the visibility of the droplets. Corresponding movie available in the ESI. Pressure profile: $p_{\mathrm{d}}=28 \mathrm{mbar}, p_{\mathrm{c}}=130 \mathrm{mbar}, \Delta p_{\mathrm{d} \text {,form }}=160 \mathrm{mbar}$, $\Delta p_{\mathrm{c}, \text { form }}=85 \mathrm{mbar}, \Delta t_{\text {form }}=0.6 \mathrm{~s}, \Delta t_{\text {pause }}=45 \mathrm{~s}, \Delta p_{\text {d, release }}=0$ mbar, $\Delta p_{\text {c,releas }}=800 \mathrm{mbar}, \Delta t_{\text {release }}=15 \mathrm{~s}, \Delta t_{\text {cycle }}=15 \mathrm{~s}$. Scale bar: $100 \mu \mathrm{m}$. 
scale out the DoD generator, we fabricated a device with 64 parallel DoD generators by further branching out the feed channels of the device with 8 parallel DoD generators. Fig. 6 illustrates the operation of this device based on four snapshots taken: before applying the first pressure pulse that initiates formation, immediately after this pulse, before applying the second pressure pulse that initiates release, and immediately after this pulse. Before the first pulse, the interfaces in all 64 generators are steadily pressed against the nozzle as shown in Fig. 6(a). While the dynamics of filling is clearly different for all generators (Fig. 6(b)), the final result, a full chamber, is the same (Fig. 6(c)). Despite differences in the speed at which the droplets are released from the chambers (evident from Fig. 6(d)), the resulting droplet volume is similar to the volume of the chamber. More quantitatively, the volumes of the droplets generated in a single DoD cycle were between $91 \%$ and $105 \%$ of the chamber volume for all 64 generators with a CoV of 0.03 for the 64 droplets. This $\mathrm{CoV}$ is comparable to that reported for common microfluidic droplet generation methods. ${ }^{40}$ To identify whether the variation mainly occurs during formation or during release, we also determined the variation in the images before release (Fig. 6(c)). The CoV (based on the area) is 0.02 , which indicates that most of the variation exists already after filling the chamber. This may be further reduced by increasing the contrast between the chamber height and channel height.

Further scale out beyond 64 generators is certainly possible. One point of attention is the footprint of the chip, which in this work has not been optimized for large scale integration purposes. The footprint can be significantly reduced by incorporating all feed channels into a separate distribution layer. With the footprint of a single DoD generator and its downstream channel being about $10 \mathrm{~mm}^{2}$, we expect that about 500 DoD generators can be comfortably fitted onto a 4 " wafer. A second point of attention is the required operating pressure, which in the current design increases with the number of DoD generators. Redesigning the feed channels based on the design rules developed earlier $^{49,58,59}$ may significantly reduce this dependency.

\section{Application of droplet on-demand: reagent supply in a droplet-based assay}

In order to show the possible use of our novel DoD generator as a tool to enable the intermittent supply of reagents/ nutrients in droplet-based assays, we fabricated a device which combines creation, storage and time-lapsing of a droplet with the intermittent supply of reagents to this droplet. The device consists of two DoD generators in series and a cup shaped trap in the downstream channel in which a droplet can be stored. First a 'mother' droplet was created using the first DoD generator. As the volume of the chamber of this generator is much smaller than the volume of the trap, the mother droplet was generated by producing a series of demi water droplets and collecting them in the trap, where (a)
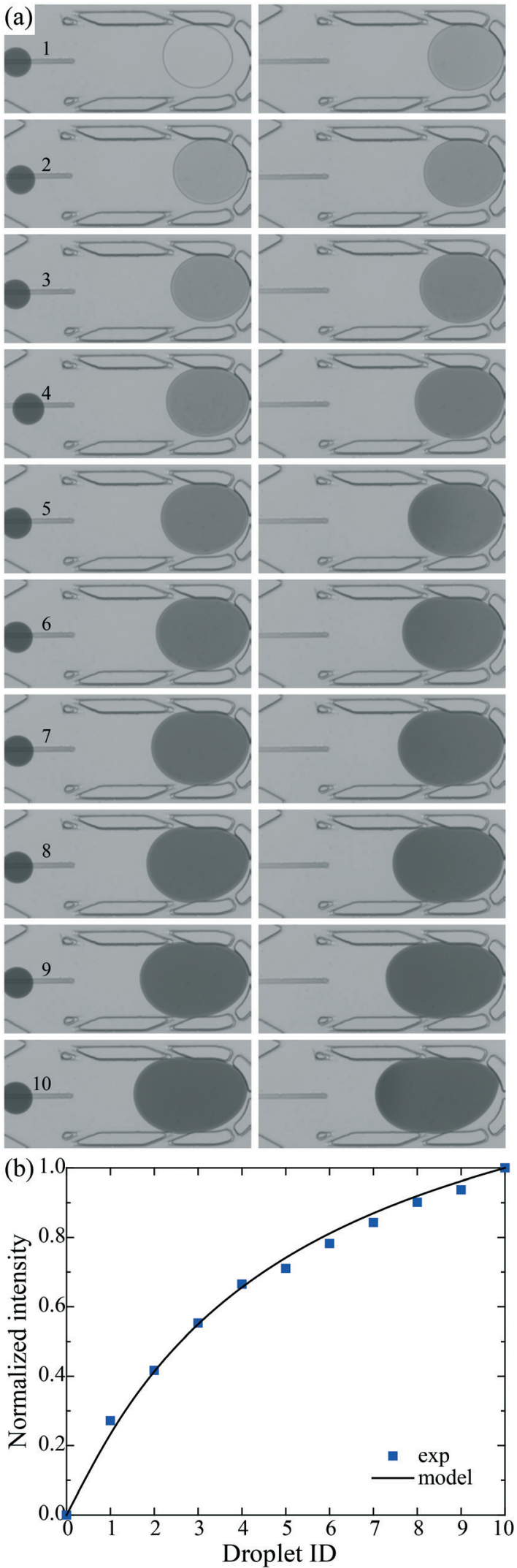

Fig. 7 Illustration of the use of the DoD generator in a simple dropletbased assay. (a) Snapshots showing the periodic addition of reagent droplets (containing methylene blue dye) to a droplet stored in a trap on a chip, before arrival (left) and after induced coalescence (right). Scale bar: $100 \mu \mathrm{m}$. (b) Relative change in the intensity of the droplet stored in the trap with increasing number of fed reagent droplets. 
they were merged. As the interfaces of the droplets were stabilized by surfactants, we coalesce them by temporarily flowing a poor solvent, perfluorooctanol, around the trap. This solvent was delivered from a separate inlet and temporary injection was controlled by elevating the pressure in the feed reservoir using the same pressure pump used to control the feed of the other fluids. After creation of the mother droplet, the second DoD generator was used for the on-demand generation of aqueous droplets containing methylene blue dye. Each formation and release cycle was followed by a temporal injection of perfluorooctanol to induce coalescence between the incoming dye droplet and stored mother droplet. The greyscale micrographs of this experiment are shown in Fig. 7(a) for a supply of 10 subsequent dye droplets. The images on the left show high consistency in the volume of the incoming reagent droplets, while the images on the right show how the mother droplet increases in volume and dye concentration. For reagent droplets of size $V_{\mathrm{r}}$ and dye concentration $c_{\mathrm{r}}$, the volume of the mother droplet increases from its initial volume $V_{\mathrm{mo}}$ to $V_{\mathrm{m}}=V_{\mathrm{m} 0}+n V_{\mathrm{r}}$, with $n$ being the number of added reagent droplets. The amount of dye added to the mother droplet increases as $n V_{\mathrm{r}} c_{\mathrm{r}}$, such that the dye concentration increases as $c_{\mathrm{m}}=c_{\mathrm{r}} n V_{\mathrm{r}} /\left(V_{\mathrm{mo}}+n V_{\mathrm{r}}\right)$. To compare this simple relation with the experiments, we measured the intensity $I$ of the mother droplet after the addition of each reagent droplet and normalised it with the initial value $\left(I_{\max }\right.$, no addition of reagent droplets) and the final value $\left(I_{\min }, 10\right.$ added reagent droplets) according to $\left(I_{\max }-I\right) /\left(I_{\max }-I_{\min }\right)$. The experiments agree well with this simple model as shown in Fig. 7(b).

The successful on-demand supply of reagents demonstrated in this relatively simple droplet-based assay shows the potential of the here developed DoD generator. Encapsulation of cells inside the mother droplet and using cell media instead of dye is a straightforward extension and opens the door to long term cell culture under semicontinuous conditions as often encountered in biotechnology at an industrial scale. The DoD approach developed in this work enables precise temporal control over bio-chemical processes studied inside droplets on a chip, making it possible to initiate, sustain, or quench processes, while monitoring them for long periods of time.

\section{Conclusions}

We presented a droplet on-demand generator that allows one to produce droplets of a predesigned volume using a commercially available pressure pump. The innovative design based on two Laplace pressure barriers enables droplets to first fill a chamber before releasing them into the main channel. This decoupling strategy ensures that the resulting volume of a droplet is dictated by the volume of the chamber and relatively insensitive to fluid properties for full wetting systems. In addition, the droplet volume is insensitive to operating conditions, which is of key importance for the scale out of DoD generators as the conditions near each generator may differ, for example due to fabrication tolerances. We demonstrated the superior performance of our DoD design in a scaled-out device with 8 DoD generators by comparing devices with and without chambers. We also demonstrated the further scale out to a device with 64 DoD generators, all producing droplets with a volume within 91\% and 105\% of the chamber volume with a $\mathrm{CoV}$ of 0.03 . We developed this scalable DoD approach to be used in droplet-based assays. Such assays require the sequential supply of reagents/ nutrients to droplets in which a process of interest is studied. We successfully demonstrated the use of the here developed DoD generator to intermittently feed reagent droplets to a droplet stored on a chip, illustrating its potential as a tool to further advance the development of droplet-based assays.

\section{Conflicts of interest}

There are no conflicts to declare.

\section{Acknowledgements}

We wish to thank Thorben de Riese and Alexander Lewis for their help with the experiments. V. v. S. is supported by a Veni grant (13137) from the Dutch Organisation for Scientific Research (NWO).

\section{Notes and references}

1 E. Brouzes, M. Medkova, N. Savenelli, D. Marran, M. Twardowski, J. B. Hutchison, J. M. Rothberg, D. R. Link, N. Perrimon and M. L. Samuels, Proc. Natl. Acad. Sci. U. S. A., 2009, 106, 14195-14200.

2 J. J. Agresti, E. Antipov, A. R. Abate, K. Ahn, A. C. Rowat, J.-C. Baret, M. Marquez, A. M. Klibanov, A. D. Griffiths and D. A. Weitz, Proc. Natl. Acad. Sci. U. S. A., 2010, 107, 4004-4009.

3 B. L. Wang, A. Ghaderi, H. Zhou, J. Agresti, D. A. Weitz, G. R. Fink and G. Stephanopoulos, Nat. Biotechnol., 2014, 32, 473.

4 T. Beneyton, I. P. M. Wijaya, P. Postros, M. Najah, P. Leblond, A. Couvent, E. Mayot, A. D. Griffiths and A. Drevelle, Sci. Rep., 2016, 6, 27223.

5 M. Huang, Y. Bai, S. L. Sjostrom, B. M. Hallström, Z. Liu, D. Petranovic, M. Uhlén, H. N. Joensson, H. Andersson-Svahn and J. Nielsen, Proc. Natl. Acad. Sci. U. S. A., 2015, 112, E4689-E4696.

6 M. Courtney, X. Chen, S. Chan, T. Mohamed, P. P. Rao and C. L. Ren, Anal. Chem., 2016, 89, 910-915.

7 K. Churski, T. S. Kaminski, S. Jakiela, W. Kamysz, W. Baranska-Rybak, D. B. Weibel and P. Garstecki, Lab Chip, 2012, 12, 1629-1637.

8 K. Leung, H. Zahn, T. Leaver, K. M. Konwar, N. W. Hanson, A. P. Pagé, C.-C. Lo, P. S. Chain, S. J. Hallam and C. L. Hansen, Proc. Natl. Acad. Sci. U. S. A., 2012, 109, 7665-7670.

9 S. H. Jin, H.-H. Jeong, B. Lee, S. S. Lee and C.-S. Lee, Lab Chip, 2015, 15, 3677-3686.

10 M. M. K. Hansen, L. H. H. Meijer, E. Spruijt, R. J. M. Maas, M. V. Rosquelles, J. Groen, H. A. Heus and W. T. S. Huck, Nat. Nanotechnol., 2015, 11, 191-197. 
11 F. Fanalista, A. Birnie, R. Maan, F. Burla, K. Charles, G. Pawlik, S. Deshpande, G. H. Koenderink, M. Dogterom and C. Dekker, ACS Nano, 2019, 13, 5439-5450.

12 L.-Y. Chu, A. S. Utada, R. K. Shah, J.-W. Kim and D. A. Weitz, Angew. Chem., Int. Ed., 2007, 46, 8970-8974.

13 L. Adams, T. E. Kodger, S.-H. Kim, H. C. Shum, T. Franke and D. A. Weitz, Soft Matter, 2012, 8, 10719-10724.

14 S. Mytnyk, I. Ziemecka, A. G. Olive, J. W. M. van der Meer, K. A. Totlani, S. Oldenhof, M. T. Kreutzer, V. van Steijn and J. H. van Esch, RSC Adv., 2017, 7, 11331-11337.

15 F. He, M.-J. Zhang, W. Wang, Q.-W. Cai, Y.-Y. Su, Z. Liu, Y. Faraj, X.-J. Ju, R. Xie and L.-Y. Chu, Adv. Mater. Technol., 2019, 1800687.

16 A. D. Griffiths and D. S. Tawfik, Trends Biotechnol., 2006, 24, 395-402.

17 B. Kintses, L. D. van Vliet, S. R. Devenish and F. Hollfelder, Curr. Opin. Chem. Biol., 2010, 14, 548-555.

18 S. L. Anna and H. C. Mayer, Phys. Fluids, 2006, 18, 121512.

19 P. Garstecki, M. J. Fuerstman, H. A. Stone and G. M. Whitesides, Lab Chip, 2006, 6, 437-446.

20 P. M. Korczyk, V. van Steijn, S. Blonski, D. Zaremba, D. A. Beattie and P. Garstecki, Nat. Commun., 2019, 10, 2528.

21 P. Abbyad, R. Dangla, A. Alexandrou and C. N. Baroud, Lab Chip, 2011, 11, 813-821.

22 A. R. Abate, T. Hung, P. Mary, J. J. Agresti and D. A. Weitz, Proc. Natl. Acad. Sci. U. S. A., 2010, 107, 19163-19166.

23 X. Niu, F. Gielen, J. B. Edel and A. J. Demello, Nat. Chem., 2011, 3, 437.

24 I. Akartuna, D. M. Aubrecht, T. E. Kodger and D. A. Weitz, Lab Chip, 2015, 15, 1140-1144.

25 D. Link, S. L. Anna, D. Weitz and H. A. Stone, Phys. Rev. Lett., 2004, 92, 054503.

26 J. Pan, A. L. Stephenson, E. Kazamia, W. T. Huck, J. S. Dennis, A. G. Smith and C. Abell, Integr. Biol., 2011, 3, 1043-1051.

27 C. H. Schmitz, A. C. Rowat, S. Köster and D. A. Weitz, Lab Chip, 2009, 9, 44-49.

28 A. Dewan, J. Kim, R. H. McLean, S. A. Vanapalli and M. N. Karim, Biotechnol. Bioeng., 2012, 109, 2987-2996.

29 N. Shembekar, C. Chaipan, R. Utharala and C. A. Merten, Lab Chip, 2016, 16, 1314-1331.

30 L. Mazutis, J. Gilbert, W. L. Ung, D. A. Weitz, A. D. Griffiths and J. A. Heyman, Nat. Protoc., 2013, 8, 870.

31 T. S. Kaminski, O. Scheler and P. Garstecki, Lab Chip, 2016, 16, 2168-2187.

32 S. Jakiela, T. S. Kaminski, O. Cybulski, D. B. Weibel and P. Garstecki, Angew. Chem., Int. Ed., 2013, 52, 8908-8911.

33 H.-H. Jeong, S. H. Jin, B. J. Lee, T. Kim and C.-S. Lee, Lab Chip, 2015, 15, 889-899.

34 H.-H. Jeong, B. Lee, S. H. Jin, S.-G. Jeong and C.-S. Lee, Lab Chip, 2016, 16, 1698-1707.
35 M. Sesen, T. Alan and A. Neild, Lab Chip, 2017, 17, 2372-2394.

36 J. Xu and D. Attinger, J. Micromech. Microeng., 2008, 18, 065020.

37 K. Churski, P. Korczyk and P. Garstecki, Lab Chip, 2010, 10, 816-818.

38 H. Zhou and S. Yao, Microfluid. Nanofluid., 2014, 16, 667-675.

39 Z. Z. Chong, S. H. Tan, A. M. Gañán-Calvo, S. B. Tor, N. H. Loh and N.-T. Nguyen, Lab Chip, 2016, 16, 35-58.

40 P. Zhu and L. Wang, Lab Chip, 2017, 17, 34-75.

41 J. Guzowski, P. M. Korczyk, S. Jakiela and P. Garstecki, Lab Chip, 2011, 11, 3593-3595.

42 U. Tangen, A. Sharma, P. Wagler and J. S. McCaskill, Biomicrofluidics, 2015, 9, 014119.

43 G. T. Vladisavljević, N. Khalid, M. A. Neves, T. Kuroiwa, M. Nakajima, K. Uemura, S. Ichikawa and I. Kobayashi, Adv. Drug Delivery Rev., 2013, 65, 1626-1663.

44 T. Kawakatsu, Y. Kikuchi and M. Nakajima, J. Am. Oil Chem. Soc., 1997, 74, 317-321.

45 M. Sun, S. S. Bithi and S. A. Vanapalli, Lab Chip, 2011, 11, 3949-3952.

46 P. M. Korczyk, L. Derzsi, S. Jakieła and P. Garstecki, Lab Chip, 2013, 13, 4096-4102.

47 V. van Steijn, P. M. Korczyk, L. Derzsi, A. R. Abate, D. A. Weitz and P. Garstecki, Biomicrofluidics, 2013, 7, 024108.

48 R. Dangla, S. C. Kayi and C. N. Baroud, Proc. Natl. Acad. Sci. U. S. A., 2013, 110, 853-858.

49 E. Amstad, M. Chemama, M. Eggersdorfer, L. R. Arriaga, M. P. Brenner and D. A. Weitz, Lab Chip, 2016, 16, 4163-4172.

50 W. Postek, T. Kaminski and P. Garstecki, Analyst, 2017, 142, 2901-2911.

51 A. M. Nightingale, G. W. Evans, P. Xu, B. J. Kim, S.-U. Hassan and X. Niu, Lab Chip, 2017, 17, 1149-1157.

52 W. Du, L. Li, K. P. Nichols and R. F. Ismagilov, Lab Chip, 2009, 9, 2286-2292.

53 F. Gielen, L. van Vliet, B. T. Koprowski, S. R. Devenish, M. Fischlechner, J. B. Edel, X. Niu, A. J. deMello and F. Hollfelder, Anal. Chem., 2013, 85, 4761-4769.

54 M. Yamada and M. Seki, Anal. Chem., 2004, 76, 895-899.

55 D. C. Duffy, J. C. McDonald, O. J. A. Schueller and G. M. Whitesides, Anal. Chem., 1998, 70, 4974-4984.

56 M. Musterd, V. van Steijn, C. R. Kleijn and M. T. Kreutzer, RSC Adv., 2015, 5, 16042-16049.

57 G. Tetradis-Meris, D. Rossetti, C. Pulido de Torres, R. Cao, G. Lian and R. Janes, Ind. Eng. Chem. Res., 2009, 48, 8881-8889.

58 M. B. Romanowsky, A. R. Abate, A. Rotem, C. Holtze and D. A. Weitz, Lab Chip, 2012, 12, 802-807.

59 S. Yadavali, H.-H. Jeong, D. Lee and D. Issadore, Nat. Commun., 2018, 9, 1222. 